\title{
Relação entre deciduidade e concentrações foliares de nutrientes em espécies lenhosas do cerrado
}

\author{
JANAINA FERNANDES DE ARAÚJO', ${ }^{1,2}$ e MUNDAYATAN HARIDASAN ${ }^{1}$
}

(recebido: 18 de janeiro de 2007; aceito: 27 de setembro de 2007)

\begin{abstract}
Correlation between deciduousness and foliar nutrient concentrations in the woody species of "cerrado"). The main objective of the present study was to compare the variations in leaf nutrient concentrations among 15 woody species of a "cerrado" sensu stricto belonging to different phenological groups to determine the influence of deciduousness on mineral nutrition. The main hypothesis was that evergreen species would present lower foliar concentrations, less specific leaf area, and smaller seasonal variations than deciduous species, because their leaves would persist beyond one annual cycle of dry and wet season. The study was conducted in a "cerrado" sensu stricto at the "Reserva Ecológica do IBGE" in the Federal District of Brazil. Fully expanded leaves collected from three individuals of each species at two-month intervals for a year were analyzed. Specific leaf area was also determined. The concentrations of $\mathrm{N}, \mathrm{P}, \mathrm{K}, \mathrm{Ca}$ and $\mathrm{Mg}$ and seasonal variation were lower in the evergreen species than in the deciduous species. Deciduous species showed higher concentrations of N, P and $\mathrm{K}$ at the beginning of the rainy season when leaves were recently formed and their concentrations decreased toward the beginning of the next dry season. Concentrations of $\mathrm{Ca}$ increased toward the end of the rainy season. Brevideciduous species had lower concentrations of $\mathrm{N}$ and $\mathrm{P}$ than deciduous species and higher concentrations of $\mathrm{N}, \mathrm{P}$ and $\mathrm{Ca}$ than evergreens. Mean specific leaf area of evergreen species $\left(54.0 \mathrm{~cm}^{2} \mathrm{~g}^{-1}\right)$ was smaller than that of deciduous $\left(67.0 \mathrm{~cm}^{2} \mathrm{~g}^{-1}\right)$ and brevideciduous species $(83.5$ $\mathrm{cm}^{2} \mathrm{~g}^{-1}$ ). Specific leaf area was correlated with $\mathrm{N}$ and $\mathrm{P}$. Thus there were significant differences not only between evergreen and deciduous species but also between deciduous and brevideciduous species.
\end{abstract}

Key words - "cerrado", mineral nutrition, specific leaf area

RESUMO - (Relação entre deciduidade e concentrações foliares de nutrientes em espécies lenhosas do cerrado). O principal objetivo deste estudo foi comparar as variações nas concentrações foliares de nutrientes de 15 espécies lenhosas do cerrado sensu stricto pertencentes a diferentes grupos fenológicos para determinar a influência da deciduidade na nutrição mineral. As principais hipóteses foram que espécies sempre-verdes apresentariam menores concentrações de nutrientes foliares, menor área foliar específica e menor variação sazonal que espécies decíduas porque suas folhas persistem por mais de um ciclo anual de estações seca e chuvosa. O estudo foi realizado em um cerrado sensu stricto na Reserva Ecológica do IBGE no Distrito Federal. Folhas completamente expandidas coletadas de três indivíduos de cada espécie, em intervalos de dois meses durante um ano, foram analisadas. A área foliar específica também foi determinada. A concentração de N, P, K, Ca e Mg e a variação sazonal foram menores nas espécies sempre-verdes do que nas decíduas. Espécies decíduas mostraram as maiores concentrações de N, P e K no inicio da estação chuvosa, época em que as folhas encontram-se recentemente maduras e suas concentrações decresceram até o começo da próxima estação seca. Concentrações de Ca foram maiores no final da estação seca. Espécies brevidecíduas tiveram menores concentrações de $\mathrm{N}$ e P que espécies decíduas e maiores concentrações de N, P, Ca e Mg que as sempre-verdes. A área foliar específica média das espécies sempre-verdes $\left(54,0 \mathrm{~cm}^{2} \mathrm{~g}^{-1}\right)$ foi menor do que a das decíduas $\left(67,0 \mathrm{~cm}^{2} \mathrm{~g} \mathrm{~g}^{-1}\right)$ e brevidecíduas $\left(83,5 \mathrm{~cm}^{2} \mathrm{~g}^{-1}\right)$. A área foliar específica foi correlacionada com $\mathrm{Ne}$ P. Houve diferenças não só entre espécies decíduas e sempre-verdes, mas também entre espécies decíduas e brevidecíduas.

Palavras-chave - área foliar específica, cerrado, nutrição mineral

\section{Introdução}

A concentração de nutrientes foliares é um importante indicador do estado de nutrição mineral de espécies lenhosas. O bioma cerrado apresenta grande diversidade e heterogeneidade de espécies mesmo em fisionomias homogêneas como o cerrado sensu stricto. Esta diversidade se reflete em espécies nativas, que

1. Universidade de Brasília, Departamento de Ecologia, Caixa postal 04457, 70904-970 Brasilia DF, Brasil.

2. Autor para correspondência: janainaf17@yahoo.com.br crescem sobre condições edáficas uniformes, pertencerem a diferentes grupos funcionais.

Desta maneira, as espécies lenhosas do cerrado apresentam padrões contrastantes de produção e perda de folhas que resultam em diferentes grupos fenológicos, constituindo assim espécies decíduas, brevidecíduas e sempre-verdes (Lenza \& Klink 2006). As espécies decíduas e brevidecíduas perdem todas as suas folhas antes do desenvolvimento de folhas novas e as sempreverdes mantém uma copa verde durante o ano todo. As espécies brevidecíduas ficam totalmente sem folhas por um curto período de tempo (menos que três semanas) e 
as decíduas permanecem sem folhas em um período de três a seis semanas. A expansão de novas folhagens nas espécies decíduas e brevidecíduas ocorre no fim da estação seca. As espécies sempre-verdes possuem uma maior longevidade foliar, sendo que suas folhas permanecem por no mínimo um ano (Franco et al. 2005). Entretanto, ocorrem variações no comportamento fenológico entre indivíduos de uma mesma população que dificultam a caracterização fenológica de algumas espécies (Lenza \& Klink 2006). Estas variações podem ocorrer também em resposta a condições edáficas ou climáticas e podem representar a plasticidade fenotípica das espécies.

A perda anual de folhas pode afetar o estado nutricional e consequentemente as funções das folhas, tais como fotossíntese e uso de água e processos como ciclagem de nutrientes.

No cerrado, ainda não foram realizados muitos estudos que correlacionem deciduidade com concentração de nutrientes foliares. Franco et al. (2005) encontraram diferença significativa na concentração de $\mathrm{N}$ e $\mathrm{P}$ foliar entre espécies decíduas e sempre-verdes e variação sazonal, sendo a maior concentração foliar de $\mathrm{N}$ e $\mathrm{P}$ encontrada nas folhas maduras recentes das plantas decíduas no fim da estação seca e tais diferenças de $\mathrm{N}$ e P nos grupos fenológicos decaíram durante a estação chuvosa.

Estudos anteriores sobre nutrição mineral de espécies nativas do cerrado foram geralmente focados em comparações entre fisionomias, na influência dos fatores edáficos e em aspectos específicos tais como acumulação de alumínio (Haridasan 1987, 1994, Haridasan \& Araújo, 2005).

A área foliar específica, que expressa a razão entre a área e massa seca da folha, reflete um "trade-off" fundamental no funcionamento de plantas entre uma rápida produção de biomassa e uma eficiente conservação de nutrientes (Poorter \& Garnier 1999). A área foliar específica é importante para entender relações hídricas da vegetação e os ciclos de carbono porque descreve a alocação da biomassa da folha por unidade de área da folha. A espessura foliar exerce uma importante função no funcionamento da folha e da planta, sendo relatada como estratégia das espécies para aquisição de recursos (Vile et al. 2005). Alguns estudos em diferentes tipos de vegetação têm mostrado uma relação positiva entre área foliar específica, conteúdo de $\mathrm{N}$ foliar e capacidade fotossintética (Meziane \& Shipley 2001, Wright et al. 2001, Hoffmann et al. 2005). Franco et al. (2005) encontrou que espécies decíduas do cerrado produzem folhas com maior área foliar específica do que espécies sempre-verdes.
Villar \& Merino (2001), quando estudaram 162 espécies lenhosas de ecossistemas contrastantes (de deserto à floresta tropical úmida) encontraram que o custo de construção foliar médio por unidade de massa seca em espécies decíduas foi significativamente mais baixo que das espécies brevidecíduas e sempre-verdes. As folhas de espécies sempre-verdes mostraram baixo conteúdo de $\mathrm{N}$ e um menor tamanho de folha que as espécies decíduas, as quais mostraram uma proporção maior de custo de construção foliar dedicado à síntese de proteínas.

O principal objetivo do presente estudo foi comparar as variações na concentração de nutrientes foliares de 15 espécies lenhosas do cerrado sensu stricto pertencentes aos três grupos fenológicos, em latossolo distrófico, para determinar a influência da deciduidade na nutrição mineral. As principais hipóteses foram que espécies sempre-verdes apresentariam menores concentrações foliares de nutrientes, menor área foliar específica e menor variação sazonal que espécies decíduas porque suas folhas persistem por mais de um ciclo anual de estações seca e chuvosa.

\section{Material e métodos}

O estudo foi desenvolvido na Reserva Ecológica do IBGE, localizada no Distrito Federal, $35 \mathrm{~km}$ ao sul de Brasília. O clima é sazonal com duas estações bem definidas, uma seca e outra chuvosa. A estação chuvosa tem seu início em setembro ou outubro e seu término em abril ou maio e a precipitação média anual é de $1.500 \mathrm{~mm}$. A temperatura média anual é de $22^{\circ} \mathrm{C}$ (RECOR, 2007). Este estudo foi realizado na fitofisionomia de cerrado sensu stricto em uma área de 1130

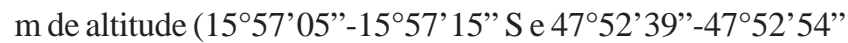
W). A área escolhida foi relativamente uniforme em relação ao ambiente edáfico, constituída por Latossolo Vermelho. As coletas de folhas e de solo foram feitas em três parcelas de $15 \times 15$ metros.

Foram estudadas 15 espécies lenhosas comuns na fitofisionomia de cerrado sensu stricto que são encontradas com facilidade na área da reserva, pertencentes aos diferentes grupos fenológicos. A classificação das espécies em decíduas, brevidecíduas e sempre-verdes está de acordo com Franco et al. (2005) e Silva Júnior (2005) (tabela 1). De maneira geral, as espécies decíduas e brevidecíduas têm sua abscisão foliar e produção de folhas novas no final da estação seca (Franco et al. 2005).

Foram feitas cinco coletas de folhas em diferentes épocas do ano (agosto e outubro de 2005, fevereiro, abril e junho de 2006) com o objetivo de contemplar a sazonalidade na concentração de nutrientes e produção de folhas. Foram coletadas folhas completamente expandidas de três indivíduos de cada espécie, com altura mínima de um metro, em cada uma das cinco coletas. As folhas coletadas foram lavadas com água destilada e secas em estufa a $70{ }^{\circ} \mathrm{C}$. O material seco 
Tabela 1. Relação de espécies lenhosas estudadas no cerrado sensu stricto da Reserva Ecológica do IBGE no Distrito Federal.

Table 1. Woody species investigated in the "cerrado" sensu stricto at the "Reserva Ecológica do IBGE" in the Federal District.

\begin{tabular}{|c|c|}
\hline Espécie & Família \\
\hline \multicolumn{2}{|l|}{ Sempre-verdes } \\
\hline Miconia pohliana Cogn. & Melastomataceae \\
\hline $\begin{array}{l}\text { Ouratea hexasperma } \\
\text { (A. St.-Hil.) Baill. }\end{array}$ & Ochnaceae \\
\hline $\begin{array}{l}\text { Schefflera macrocarpa } \\
\text { (Cham. \& Schltdl.) Frodin }\end{array}$ & Araliaceae \\
\hline Sclerolobium paniculatum Vogel & Fabaceae \\
\hline Vochysia elliptica Mart. & Vochysiaceae \\
\hline Vochysia thyrsoidea Pohl & Vochysiaceae \\
\hline \multicolumn{2}{|l|}{ Brevidecíduas } \\
\hline $\begin{array}{l}\text { Blepharocalyx salicifolius } \\
\text { (Kunth) O. Berg }\end{array}$ & Myrtaceae \\
\hline Byrsonima crassa Nied. & Malpighiaceae \\
\hline Caryocar brasiliense Cambess. & Cariocaraceae \\
\hline Dalbergia miscolobium Benth. & Fabaceae \\
\hline $\begin{array}{l}\text { Stryphnodendron adstringens } \\
\text { (Mart.) Coville. }\end{array}$ & Fabaceae \\
\hline \multicolumn{2}{|l|}{ Decíduas } \\
\hline Guapira noxia (Netto) Lundell. & Nyctaginaceae \\
\hline Kielmeyera coriacea Mart. \& Zucc. & Guttiferae \\
\hline Qualea grandiflora Mart. & Vochysiaceae \\
\hline Qualea parviflora Mart. & Vochysiaceae \\
\hline
\end{tabular}

foi moído em moinho tipo Wiley. A concentração de nitrogênio nas folhas foi determinada utilizando-se o método de digestão e destilação de micro-Kjeldahl. A determinação das concentrações de $\mathrm{P}, \mathrm{K}, \mathrm{Ca}, \mathrm{Mg}, \mathrm{Mn}, \mathrm{Zn}, \mathrm{Cu}$ e $\mathrm{Al}$ nas folhas foi feita por meio de digestão, utilizando-se uma mistura triácida de ácidos nítrico, sulfúrico e perclórico, na proporção de 10:1:2. A concentração de P no extrato foi determinada por colorimetria, a $410 \mathrm{~nm}$, utilizando-se vanadomolibdato de amônia. As concentrações dos demais nutrientes foram obtidas por espectrofotometria de absorção atômica ou emissão de chama (Allen 1989).

A área foliar específica das espécies estudadas foi definida em duas épocas do ano, abril (final da estação chuvosa) e junho (estação seca), onde as folhas se encontravam em seu estado final de crescimento. Logo após serem coletadas, as imagens das folhas foram digitalizadas através de scanner (HP PrecisionScan LTX), com resolução de 100 dpi. O arquivo conteve imagem de todas as folhas coletadas de cada indivíduo. Para as folhas compostas, os folíolos foram separados, sendo medida a área do conjunto de folíolos. A área foliar foi calculada de acordo com Caldas et al. (1992). Após secagem completa em estufa a $70{ }^{\circ} \mathrm{C}$, o conjunto de folhas de cada indivíduo foi pesado e a área foliar específica foi calculada a partir da razão entre a área foliar e massa seca de todas as folhas de cada indivíduo.

As amostragens de solo foram realizadas em dezembro de 2005, estação chuvosa, e junho de 2006, estação seca. Foram coletadas cinco amostras compostas de 0-5, 5-10, 1020 e 20-30 cm de profundidade em cada parcela. As amostras foram secas ao ar e passadas em peneira com abertura de malha de dois milímetros. Os valores de $\mathrm{pH}$ foram determinados em água e $\mathrm{KCl}(1 \mathrm{M})$, em uma suspensão solo:solução de 1:2,5. O teor de matéria orgânica e carbono orgânico foi determinado pelo método de Walkley \& Black (Allen 1989). O nitrogênio total foi determinado pelo método micro-Kjeldahl. A disponibilidade de $\mathrm{P}, \mathrm{K}, \mathrm{Mn}, \mathrm{Fe}, \mathrm{Zn}$ e $\mathrm{Cu}$ no solo foi determinada em extrato de Mehlich $\left(\mathrm{H}_{2} \mathrm{SO}_{4}, 0,0125 \mathrm{M}+\mathrm{HCl}\right.$, $0,05 \mathrm{M}$ ) e de $\mathrm{Ca}, \mathrm{Mg}$ e Al em KCl, $1 \mathrm{M}$ (Embrapa 1997). O teor de $\mathrm{P}$ no extrato de solo foi determinado por colorimetria a 660 $\mathrm{nm}$, utilizando-se o molibdato de amônia. O teor de alumínio foi determinado por titulação com $\mathrm{NaOH}$. Os teores dos elementos $\mathrm{K}, \mathrm{Ca}, \mathrm{Mg}, \mathrm{Mn}, \mathrm{Fe}, \mathrm{Zn}$ e $\mathrm{Cu}$ foram determinados por espectrofotometria de absorção atômica ou emissão de chama. (Allen 1989).

Foi utilizada análise de variância para testar diferenças entre os grupos fenológicos, espécies e épocas de coleta. Também foi utilizada análise de variância para testar as diferenças entre as profundidades do solo e as épocas de coleta. O teste de Tukey a 5\% de probabilidade foi utilizado para comparação de médias das análises de solo e de folhas através do programa SAS (2006).

\section{Resultados}

Propriedades do solo - Os valores de $\mathrm{pH}$ em água e em $\mathrm{KCl} 1 \mathrm{M}$ aumentaram com a profundidade e os valores foram mais baixos na época chuvosa do que na época seca. O teor de carbono orgânico diminuiu com a profundidade e foram maiores na estação chuvosa. $\mathrm{O}$ teor de nitrogênio diminuiu com a profundidade e os maiores valores foram encontrados na estação seca. Os teores de fósforo encontrados foram muito baixos. Os teores dos cátions trocáveis $(\mathrm{Ca}, \mathrm{Mg}$ e $\mathrm{K})$ decresceram com a profundidade e foram maiores na estação chuvosa. Os teores de alumínio foram maiores na estação seca. Os teores de ferro não apresentaram diferença significativa entre as datas de coleta. Os teores dos demais micro nutrientes foram maiores na estação chuvosa e também decresceram com a profundidade (tabela 2).

Concentração de nutrientes foliares - As espécies com deciduidade apresentaram maiores concentrações de macronutrientes foliares e do que as sempre-verdes (figura 1). Vale ressaltar que Guapira noxia, espécie decídua, 
Tabela 2. Propriedades químicas do solo do cerrado sensu stricto da Reserva Ecológica do IBGE no Distrito Federal. $n=$ 15; entre parênteses o desvio padrão. Letras minúsculas representam diferenças significativas entre profundidades, letras maiúsculas representam diferenças significativas entre a estação seca e chuvosa pelo Teste de Tukey $(P<0,05)$.

Table 2. Chemical properties of the soil of the "cerrado" sensu stricto at the "Reserva Ecológica do IBGE" in the Federal District. $n=15$; standard deviation in parenthesis. Small case letter indicates significant differences among depths and upper case letters between the rainy and dry seasons according to Tukey's test $(P<0,05)$.

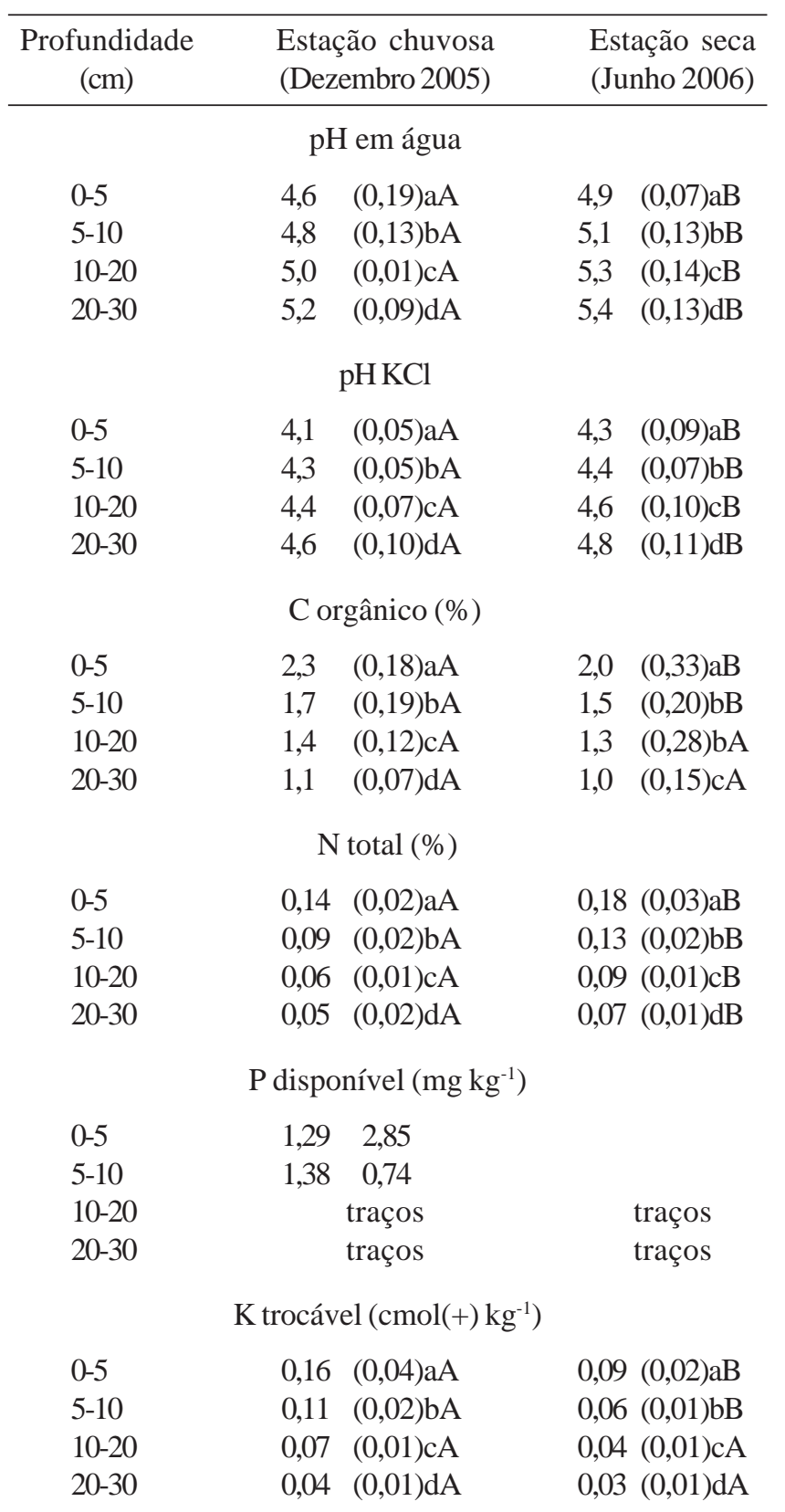

continua continuação

\begin{tabular}{ccc}
\hline $\begin{array}{c}\text { Profundidade } \\
(\mathrm{cm})\end{array}$ & $\begin{array}{c}\text { Estação chuvosa } \\
(\text { Dezembro 2005) }\end{array}$ & $\begin{array}{c}\text { Estação seca } \\
(\text { Junho 2006) }\end{array}$ \\
\hline \multicolumn{3}{c}{ Ca trocável $\left(\operatorname{cmol}(+) \mathrm{kg}^{-1}\right)$} \\
$0-5$ & $0,05(0,01) \mathrm{aA}$ & $0,03(0,01) \mathrm{aB}$ \\
$5-10$ & $0,02(0,01) \mathrm{bA}$ & $0,02(0,01) \mathrm{bA}$ \\
$10-20$ & $0,01 \quad(0,003) \mathrm{cA}$ & $0,01(0,003) \mathrm{cA}$ \\
$20-30$ & $0,01 \quad(0,003) \mathrm{cA}$ & $0,01(0,004) \mathrm{cA}$
\end{tabular}

Mg trocável $\left(\operatorname{cmol}(+) \mathrm{kg}^{-1}\right)$

$\begin{array}{llll}0-5 & 0,09 & (0,02) \mathrm{aA} & 0,04(0,01) \mathrm{aB} \\ 5-10 & 0,02 & (0,005) \mathrm{bA} & 0,03(0,01) \mathrm{bB} \\ 10-20 & 0,01 & (0,004) \mathrm{cA} & 0,02(0,01) \mathrm{cB} \\ 20-30 & 0,01 & (0,002) \mathrm{dA} & 0,02(0,01) \mathrm{dB}\end{array}$

Al trocável $\left(\operatorname{cmol}(+) \mathrm{kg}^{-1}\right)$

$\begin{array}{lllll}0-5 & 1,0 & (0,17) \mathrm{aA} & 1,1 & (0,27) \mathrm{aB} \\ 5-10 & 0,6 & (0,11) \mathrm{bA} & 0,7 & (0,17) \mathrm{bB} \\ 10-20 & 0,4 & (0,08) \mathrm{cA} & 0,4 & (0,1) \mathrm{cB} \\ 20-30 & 0,2 & (0,04) \mathrm{dA} & 0,3 & (0,07) \mathrm{dB}\end{array}$

Fe disponível $\left(\mathrm{mg} \mathrm{kg}^{-1}\right)$

$\begin{array}{lllll}0-5 & 73 & (10,4) \mathrm{aA} & 69 & (11,6) \mathrm{aB} \\ 5-10 & 64 & (11,1) \mathrm{bA} & 61 & (13,0) \mathrm{bB} \\ 10-20 & 39 & (13,6) \mathrm{cA} & 41 & (10,4) \mathrm{cB} \\ 20-30 & 31 & (7,83) \mathrm{dA} & 25 & (7,27) \mathrm{dB}\end{array}$

Mn disponível $\left(\mathrm{mg} \mathrm{kg}^{-1}\right)$

$\begin{array}{lllll}0-5 & 3,5 & (0,99) \mathrm{aA} & 2,8 & (0,88) \mathrm{aB} \\ 5-10 & 1,9 & (0,30) \mathrm{bA} & 1,6 & (0,43) \mathrm{bB} \\ 10-20 & 1,2 & (0,21) \mathrm{cA} & 1,0 & (0,24) \mathrm{cB} \\ 20-30 & 1,1 & (0,09) \mathrm{cA} & 0,7 & (0,17) \mathrm{cB}\end{array}$

Zn disponível $\left(\mathrm{mg} \mathrm{kg}^{-1}\right)$

$\begin{array}{lllll}0-5 & 1,6 & (0,33) \mathrm{aA} & 0,7 & (0,26) \mathrm{aB} \\ 5-10 & 1,3 & (0,26) \mathrm{bA} & 0,6 & (0,26) \mathrm{abB} \\ 10-20 & 0,9 & (0,20) \mathrm{bcA} & 0,7 & (0,13) \mathrm{abA} \\ 20-30 & 0,9 & (0,18) \mathrm{cA} & 0,8 & (0,15) \mathrm{bA}\end{array}$

Cu disponível ( $\left.\mathrm{mg} \mathrm{kg}^{-1}\right)$

$\begin{array}{lllll}0-5 & 0,5 & (0,11) \mathrm{abA} & 0,4 & (0,06) \mathrm{abB} \\ 5-10 & 0,6 & (0,15) \mathrm{aA} & 0,4 & (0,08) \mathrm{aB} \\ 10-20 & 0,5 & (0,08) \mathrm{abA} & 0,4 & (0,17) \mathrm{abB} \\ 20-30 & 0,5 & (0,10) \mathrm{bA} & 0,3 & (0,06) \mathrm{bB}\end{array}$

apresentou concentração foliar de N, P, K e Mg bem superiores às demais espécies. As brevidecíduas apresentaram maiores concentrações foliares de $\mathrm{Ca}$ e não houve variação significativa entre o grupo de decíduas e sempre-verdes para este nutriente (figura 1). Houve diferença significativa na concentração de 

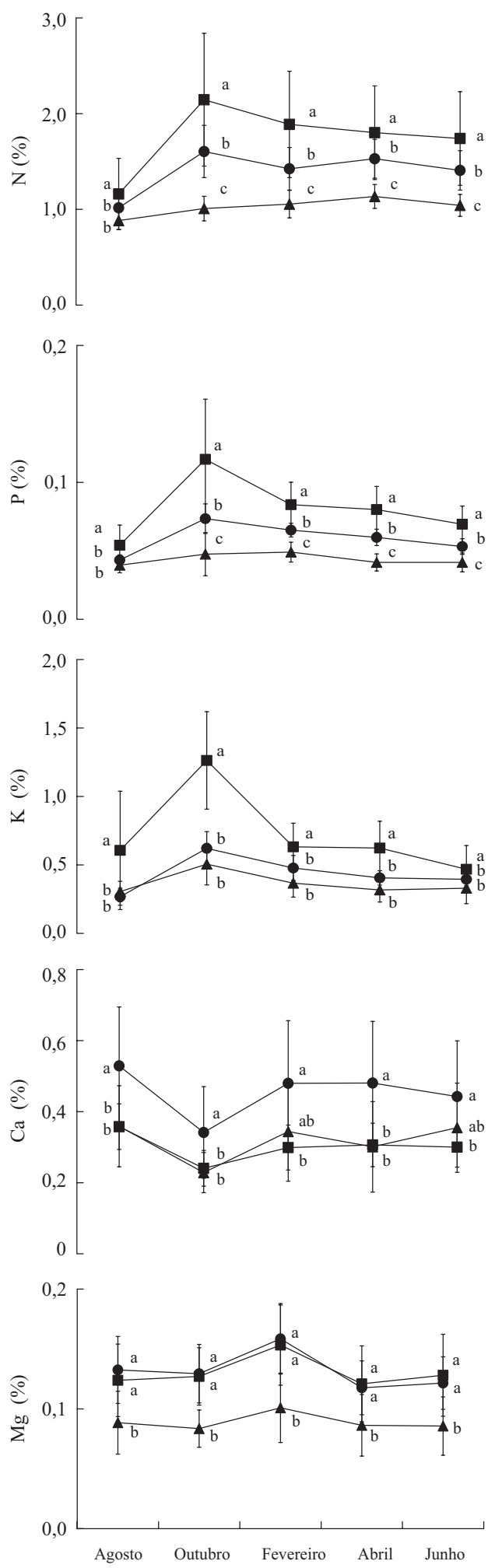

Figura 1. Variação sazonal na concentração foliar de macronutrientes entre grupos fenológicos de espécies lenhosas de um cerrado sensu stricto na Reserva Ecológica do IBGE no Distrito Federal. As barras verticais representam o desvio padrão. As letras representam diferenças significativas entre grupos fenológicos pelo Teste de Tukey $(P<0,05)$.

( $\boldsymbol{\Delta}$ = sempre-verdes; $\bullet$ = brevidecíduas; $\mathbf{\square}=$ decíduas)
$\mathrm{N}$, P e K foliar entre as decíduas e brevidecíduas, mas não houve diferença na concentração de $\mathrm{Mg}$ foliar entre esses dois grupos fenológicos. Em análise sem a inclusão da espécie Guapira noxia, as brevidecíduas apresentariam maior concentração foliar de N. A concentração de nutrientes foliares variou significativamente entre as espécies dentro de cada grupo fenológico (figura 2).

As espécies decíduas e brevidecíduas apresentaram maior concentração de N, Pe K foliar no inicio da estação chuvosa e menores concentrações desses nutrientes no final da estação seca (figura 1). As sempre-verdes, de maneira geral, não apresentaram variação sazonal de N, P e K. Em termos de espécies, a variação da concentração de N, P e K foliar entre as datas de coleta é maior para as espécies decíduas e brevidecíduas do que para as espécies sempre-verdes. Por exemplo, a espécie decídua Kielmeyera coriacea apresentou concentração de $\mathrm{K}$ foliar igual a $0,16 \%$ no mês de agosto e $1,27 \%$ no mês de outubro enquanto a sempre-verde Miconia pohliana apresentou concentração de $\mathrm{K}$ variando entre $0,22 \%$ e $0,32 \%$ nos meses de abril e junho. A concentração de Ca foliar para os três grupos fenológicos foi menor no inicio da estação chuvosa, apresentando padrão de variação sazonal, de maneira geral, diferente dos demais nutrientes, pois algumas espécies apresentaram maior concentração desse elemento no mês de agosto (dados não apresentados). A concentração de $\mathrm{Mg}$ foliar foi maior durante o mês de fevereiro (meio da estação chuvosa) também para os três grupos fenológicos.

Área foliar específica - A média ( \pm desvio padrão) de área foliar específica de todas as espécies lenhosas do cerrado sensu stricto estudado foi $67,3( \pm 19,8) \mathrm{cm}^{2} \mathrm{~g}^{-1}$ variando de 33,8 $( \pm 2,54)$ a $111( \pm 9,52) \mathrm{cm}^{2} \mathrm{~g}^{-1}$. A espécie que apresentou maior área foliar específica foi a brevidecídua Dalbergia miscolobium, e o menor valor foi apresentado pela sempre-verde Schefflera macrocarpa (figura 3). A área foliar especifica média do grupo de brevidecíduas foi a maior $\left(83,5 \pm 19,2 \mathrm{~cm}^{2} \mathrm{~g}^{-1}\right)$, seguido pelo grupo de decíduas $\left(67 \pm 12 \mathrm{~cm}^{2} \mathrm{~g}^{-1}\right)$. A menor área foliar específica foi encontrada no grupo de sempre-verdes $\left(54 \pm 13,7 \mathrm{~cm}^{2} \mathrm{~g}^{-1}\right)$ (figura 4). Não foram observadas diferenças significativas de área foliar específica entre

Figure 1. Seasonal variation in the foliar concentration of macronutrients among phenological groups of woody species in a "cerrado" sensu stricto at the "Reserva Ecológica do IBGE" in the Federal District. Vertical bars represent standard deviation. Small case letters indicate differences among phenological groups by Tukey's test $(P<0.05)$.

( $\boldsymbol{\Lambda}$ = evergreen; $\bullet=$ brevideciduous; $\mathbf{\square}=$ deciduous $)$ 

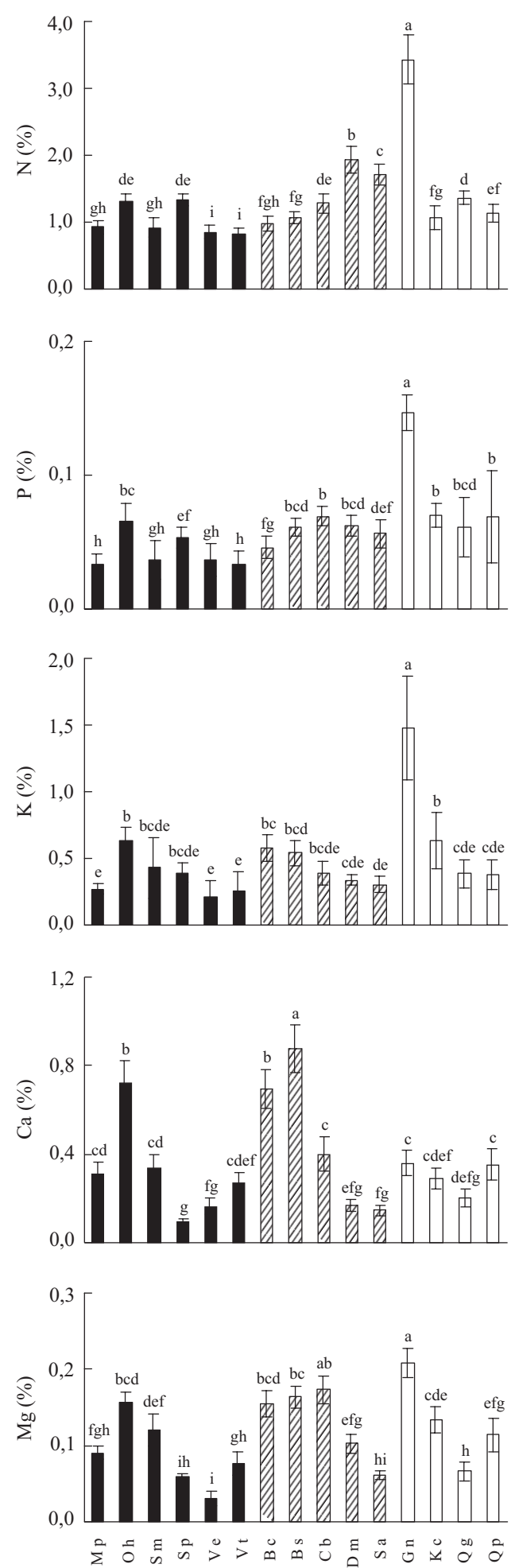

Figura 2. Variação na concentração foliar de macronutrientes entre espécies lenhosas de um cerrado sensu stricto na Reserva Ecológica do IBGE no Distrito Federal. A primeira letra indica o gênero e a segunda a espécie conforme tabela 1 . As barras verticais representam o desvio padrão. As letras representam diferenças entre as médias das concentrações foliares dos nutrientes pelo Teste de Tukey $(P<0,05)$.

( $\mathbf{\square}$ = sempre-verdes; $\mathbb{Z}=$ brevidecíduas; $\square=$ decíduas)

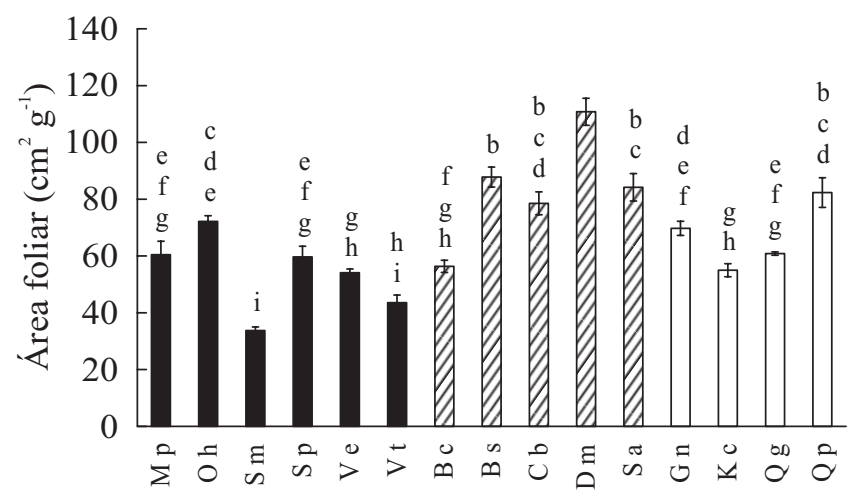

Figura 3. Área foliar específica de espécies lenhosas de um cerrado sensu stricto na Reserva Ecológica do IBGE, Brasília. A primeira letra indica o gênero e a segunda a espécie conforme tabela 1. As barras representam o desvio padrão. As letras minúsculas representam diferenças significativas entre as médias das espécies, pelo teste de Tukey $(P<0,05)$.

( $\square=$ sempre-verdes; $\mathbb{\square}=$ brevidecíduas; $\square=$ decíduas)

Figure 3. Variation in specific leaf area among the woody species in a "cerrado" sensu stricto at the "Reserva Ecológica do IBGE" in the Federal District. The first letter represents the genus and the second the species as listed in Table 1. Vertical bars represent standard deviation. Small case letters indicate differences among species by Tukey's test $(P<0.05)$.

( $\square$ = evergreen; $\mathbb{\square}=$ brevideciduous; $\square=$ deciduous)

os meses de abril e junho em termos de espécies e em termos de grupos fenológicos.

Houve correlação positiva entre as concentrações de $\mathrm{N}$ e $\mathrm{P}$ nas folhas (figura 5) e entre a área específica foliar e estes nutrientes (figuras 6 e 7). Entretanto o grau de correlação foi influenciado pelo comportamento de $G$. noxia, que apresentou teores muito maiores de $\mathrm{N}$ e $\mathrm{P}$ do que as outras espécies estudadas.

\section{Discussão}

Os resultados das análises químicas do solo confirmaram a pouca disponibilidade de nutrientes e forte acidez descrita para latossolos no bioma cerrado (Lopes \& Cox 1977, Haridasan 1987, 1994, Marimon Júnior \& Haridasan, 2005). Os cátions trocáveis (Ca, Mg e K),

Figure 2. Variation in the foliar concentration of macronutrients among the woody species in a "cerrado" sensu stricto at the "Reserva Ecológica do IBGE" in the Federal District. The first letter represents the genus and the second the species as listed in Table 1. Vertical bars represent standard deviation. Small case letters indicate differences among phonological groups by Tukey's test $(P<0.05)$.

( $\square$ = evergreen; $\square=$ brevideciduous; $\square=$ deciduous $)$ 


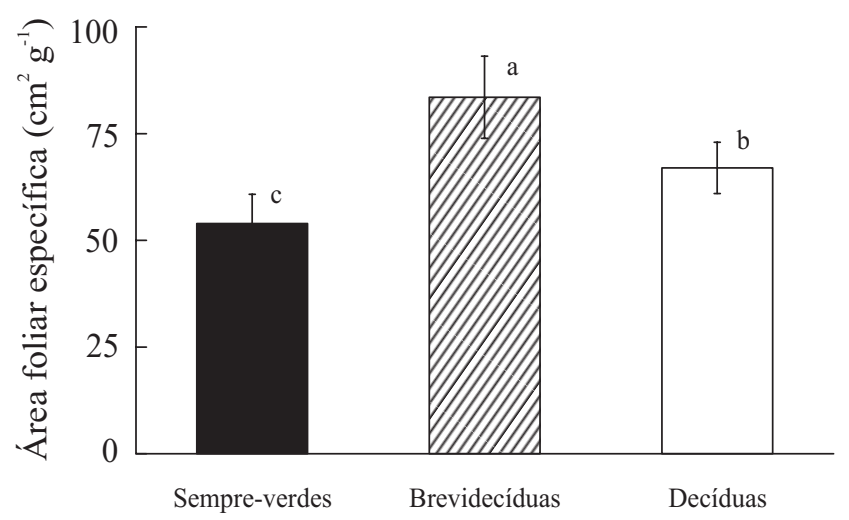

Figura 4. Variação na área foliar específica entre os grupos fenológicos. As letras minúsculas representam diferenças significativas entre grupos fenológicos, pelo teste de Tukey $(P<0,05)$. As barras representam o desvio padrão.

( $\square=$ sempre-verdes; $\square=$ brevidecíduas; $\square=$ decíduas)

Figure 4. Variation in specific leaf area among phenologic groups. Vertical bars represent standard deviation. Small case letters indicate differences among species by Tukey's test $(P<0.05)$.

( $\square=$ evergreen; $\square=$ brevideciduous; $\square=$ deciduous)

de modo geral, apresentaram maior disponibilidade na estação chuvosa, bem como os teores dos micronutrientes, talvez devido à mineralização da serapilheira, que se apresenta em maior quantidade no fim da estação seca (Peres et al. 1983).

As concentrações foliares de $\mathrm{N}$ e $\mathrm{P}$ foram correlacionadas (figura 5). Franco et al. (2005) e Nardoto et al. (2006) também encontraram correlação linear positiva entre as concentrações de $\mathrm{N}$ e $\mathrm{P}$, bem como Wright et al. (2001), que observou forte associação nas concentrações foliares de $\mathrm{N}$ e $\mathrm{P}$ em locais secos e fraca associação em locais úmidos em diferentes ecossistemas. Correlações entre $\mathrm{N}$ e $\mathrm{P}$ refletem sua associação na bioquímica da planta, particularmente na síntese de proteínas (Garten Junior 1976). Aerts \& Chapin (2000) definiram valores de N/P menores que 16 como limitação por $\mathrm{N}$, entre 14 e 16 como co-limitação por $\mathrm{N}$ e $\mathrm{P}$ e superiores à 16 como limitação por $\mathrm{P}$. A razão média de N/P encontrada no presente trabalho foi 23, indicando uma forte limitação de $\mathrm{P}$ para as espécies lenhosas estudadas, o que está associado à baixa disponibilidade de $\mathrm{P}$ encontrada nas análises de solo. Este resultado de limitação de $\mathrm{P}$ também foi observado por Nardoto et al. (2006), onde a razão N/P encontrada foi 18. Porém, quando observado os valores de N/P para cada espécie, as decíduas Kielmeyera coriacea e Qualea parviflora e as brevidecíduas Blepharocalyx salicifolius e Caryocar brasiliense apresentaram limitação por $\mathrm{N}$ no inicio da estação chuvosa.

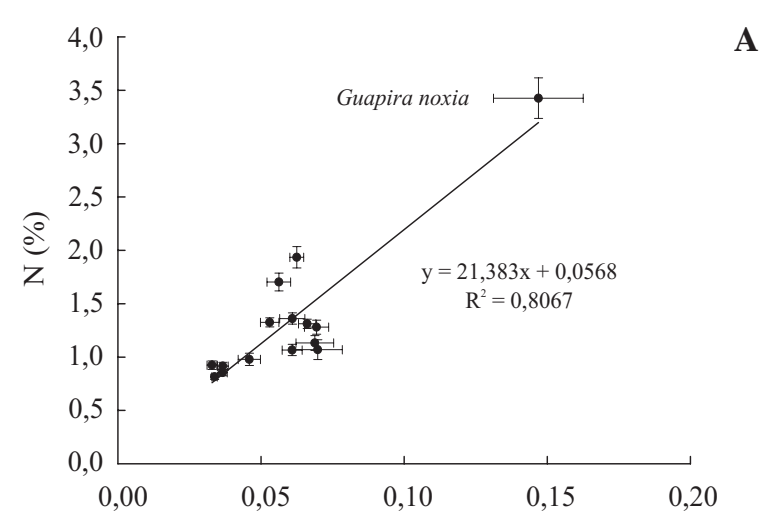

A

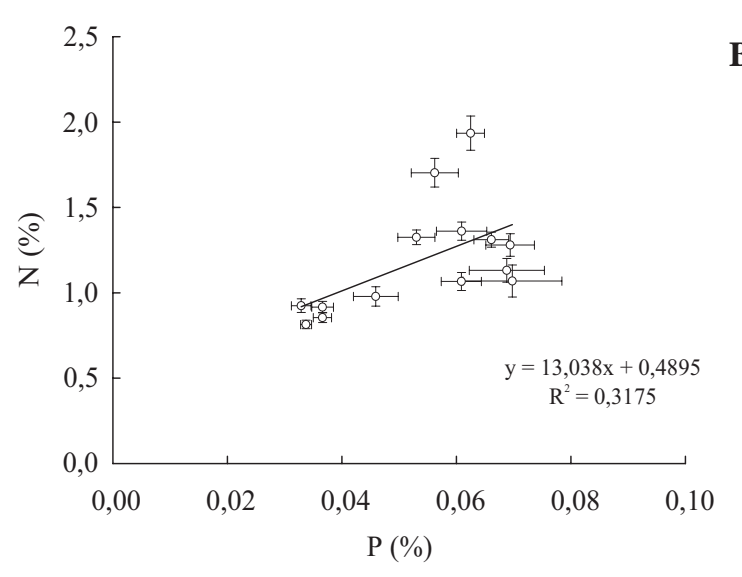

Figura 5. Correlação entre as concentrações foliares de N e P de espécies lenhosas de um cerrado sensu stricto da Reserva Ecológica do IBGE no Distrito Federal, com (A) e sem Guapira noxia (B). As barras representam o erro padrão da média $(n=15)$.

Figure 5. Correlation between foliar concentrations of $\mathrm{N}$ and $\mathrm{P}$ in the woody species in a "cerrado" sensu stricto at the "Reserva Ecológica do IBGE" in the Federal District, with (A) and without (B) the species Guapira noxia. Bars represent standard error of the mean $(n=15)$.

Vale ressaltar que a espécie nativa, Guapira noxia, apresentou concentrações de $\mathrm{N}$ e $\mathrm{P}$ foliares mais altas do que todas as outras espécies em todas as coletas (figuras 2, 5-7). Outros autores como Bustamante et al. (2004) também encontraram altos valores de N para esta espécie em área de cerrado. Não foram encontrados registros sobre fixação de nitrogênio em Guapira noxia, deixando em aberto pesquisas para investigar o fato desse gênero apresentar altas concentrações de N.

As espécies decíduas apresentaram maior concentração foliar para os macronutrientes N, P, K e $\mathrm{Mg}$ (figura 1). Villar et al. (2006), analisando espécies lenhosas de diferentes famílias, encontrou maior concentração mineral nas espécies decíduas, apesar das 

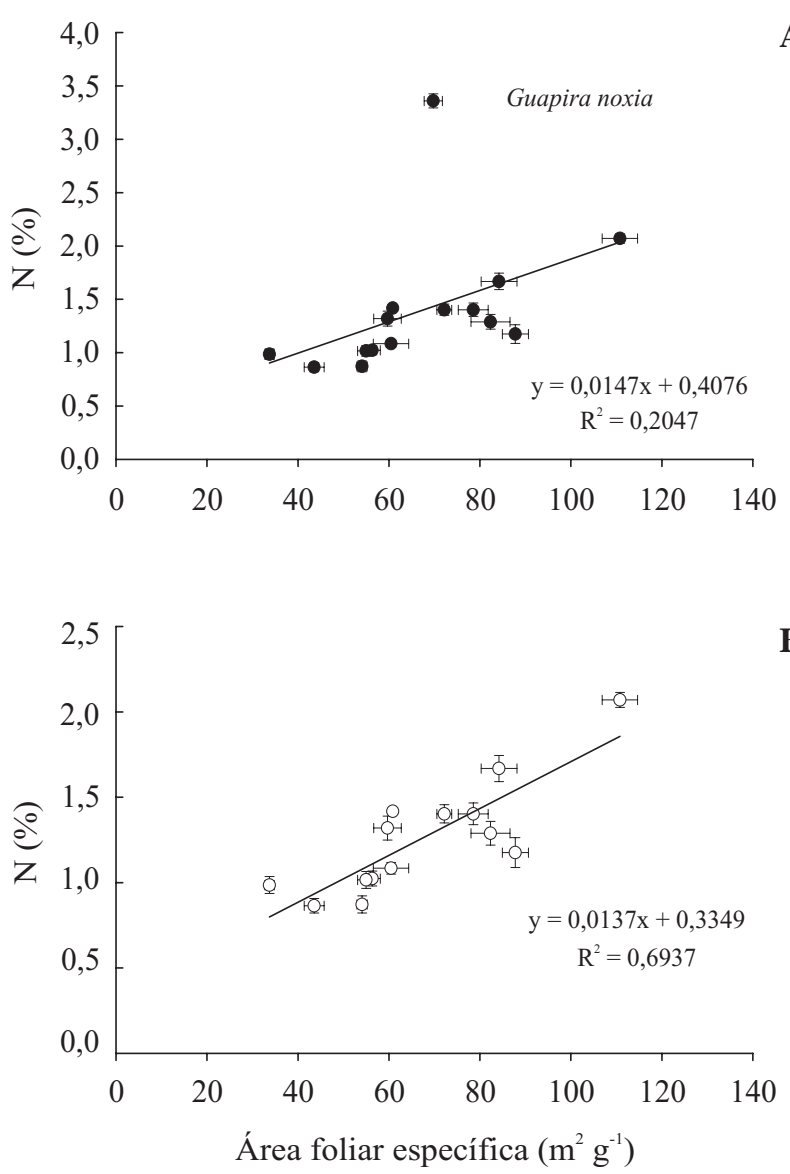

Figura 6. Correlação entre área foliar específica e a concentração foliar de $\mathrm{N}$ em espécies lenhosas de um cerrado sensu stricto da Reserva Ecológica do IBGE no Distrito Federal, com (A) e sem Guapira noxia (B). As barras representam o erro padrão da média $(n=6)$.

Figure 6. Correlation between specific leaf area and foliar concentrations of $\mathrm{N}$ in the woody species in a "cerrado" sensu stricto at the "Reserva Ecológica do IBGE" in the Federal District, with (A) and without (B) the species Guapira noxia. Bars represent standard error of the mean $(n=6)$.

diferenças entre sempre-verdes e decíduas terem sido pequenas, e encontrou que a filogenia explica em 50\% a $85 \%$ a variação na composição química das espécies concluindo que nesse caso a filogenia foi um fator mais importante que o grupo funcional para explicar composição química. As menores concentrações de $\mathrm{N}$ e $\mathrm{P}$ foliares nas espécies sempre-verdes é fato observado também por Montes \& Medina (1977), Franco et al. (2005) e Nardoto et al. (2006). A menor concentração foliar de $\mathrm{N}, \mathrm{P}, \mathrm{K}$ e Mg para as sempre-verdes tem como possível explicação a maior longevidade foliar deste grupo. As sempre-verdes não apresentaram maiores valores de concentração foliar para nenhum dos macronutrientes em comparação a espécies decíduas.
A menor concentração foliar de N, P e K, observada no mês de agosto de 2005, pode estar associada ao fato de que, nesse mês, a maioria das espécies encontra-se em época de abscisão foliar, ou seja, pode ter ocorrido translocação desses nutrientes. N, P e K são elementos moveis que podem ser facilmente retranslocados de uma parte da planta para outra, pode ser observado a baixa concentração foliar no mês de agosto, maiores concentrações em outubro, época em que as folhas estão recentemente maduras para a maioria das espécies. Já no caso da concentração foliar de $\mathrm{Ca}$, o qual é um elemento de difícil translocação, o mês de outubro apresentou os menores valores. Os resultados de sazonalidade na concentração de nutrientes mostraram que a as concentrações de nutrientes
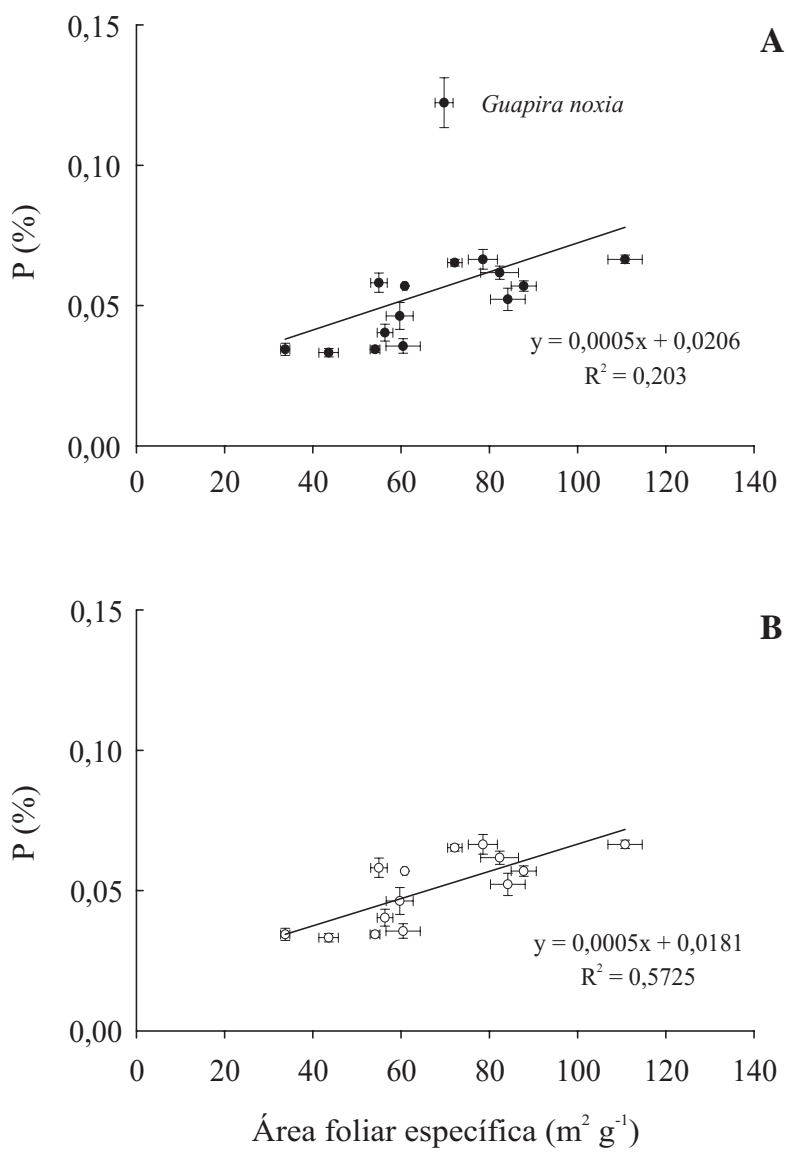

Figura 7. Correlação entre área foliar específica e a concentração foliar de $\mathrm{P}$ em espécies lenhosas de um cerrado sensu stricto da Reserva Ecológica do IBGE no Distrito Federal, com (A) e sem Guapira noxia (B). As barras representam o erro padrão da média $(n=6)$.

Figure 7. Correlation between specific leaf area and foliar concentrations of P in the woody species in a "cerrado" sensu stricto at the "Reserva Ecológica do IBGE" in the Federal District, with (A) and without (B) the species Guapira noxia. Bars represent standard error of the mean $(n=6)$. 
foliares variam ao longo de um ano de acordo com as diferenças nos padrões de produção e queda de folhas. Villela \& Lacerda (1992) concluíram de que a sazonalidade na concentração de nutrientes pode estar mais relacionada com os mecanismos de absorção e translocação dos elementos associados à idade foliar do que com variações ambientais ao longo do ano.

As espécies decíduas apresentaram maior área foliar específica do que as sempre-verdes, sendo que as brevidecíduas apresentaram os maiores valores. Este resultado está de acordo com o encontrado por Silva (2001) que analisou parâmetros foliares de espécies lenhosas de três fitofisionomias do Cerrado que formam um gradiente de vegetação (cerrado sensu stricto, cerrado denso e campo sujo) e encontrou uma menor área foliar específica para espécies sempre-verdes e maior para as espécies brevidecíduas. Reich et al. (1992) e Reich (1993) mostraram que área foliar específica tem correlação com a longevidade das folhas, onde o valor de área foliar específica decresce com o aumento da longevidade foliar. Provavelmente espécies com menor área foliar específica investem mais em massa por unidade de área, na construção foliar com maior longevidade (Reich et al. 1997, Westoby et al. 2000).

A área foliar específica foi positivamente correlacionada com N (figura 6) e P (figura 7), Este resultado de associação positiva entre área foliar específica e $\mathrm{N}$ e entre área foliar específica e $\mathrm{P}$ também foi encontrado por Wright et al. (2001) quando analisou 79 espécies em habitats com altos e baixos teores de nutrientes. As altas concentrações de N e P em Guapira noxia afetam estas correlações.

Assim concluímos que as hipóteses formuladas de que as espécies sempre verdes apresentariam menores concentrações foliares de macronutrientes e menor área foliar foram substanciadas pelos resultados obtidos. Entretanto houve diferenças significativas entre espécies decíduas e brevidecíduas nas características estudadas e as variações interespecíficas dentro de grupos fenológicos foram também as vezes maiores do que entre os grupos.

Agradecimentos - O presente estudo foi financiado pela CAPES e pela National Science Foundation como parte do projeto "Biocomplexity of hydrologically controlled vegetation dynamics: quantitative-comparative analysis of Everglades and "Cerrado" ecosystems under soil moisture and nutrient spatial-temporal dynamics". Agradecemos ao professor Lúcio José Vivaldi, pela ajuda nas análises estatísticas, e à química Mara Rúbia Suzana Chaves, pelo apoio nas análises de laboratório. Agradecemos à administração da Reserva Ecológica do IBGE, pela autorização e apoio no desenvolvimento deste trabalho.

\section{Referências bibliográficas}

AERTS, R. \& CHAPIN, F.S. 2000. The mineral nutrition of wild plants revisited: a re-evaluation of processes and patterns. Advances in Ecological Research 30:1-67.

ALLEN, S.E. 1989. Chemical analysis of ecological materials. Blackwell Scientific Publications, Oxford.

BUSTAMANTE, M.M.C., MARTINELLI, L.A., SILVA, D.A., CAMARGO, P.B., KLINK, C.A., DOMINGUES, T.F. \& SANTOS, R.V. 2004. 15N natural abundance in woody plants and soils of Central Brazilian savannas (cerrado). Ecological Applications 14 supplement:200-213.

CALDAS, L.S., BRAVO, C., PICCOLO H. \& FARIA, C.R.S.M. 1992. Measurement of leaf area with a hand-scanner linked to a microcomputer. Revista Brasileira de Fisiologia Vegetal 4:17-20.

EMBRAPA. 1997. Manual de métodos de análises do solo. Serviço Nacional de Levantamento e Conservação do Solo, Rio de Janeiro.

FRANCO, A.C., BUSTAMANTE, M., CALDAS, L.S.; GOLDSTEIN, G., MEINZER, F.C., KOZOVITS, A.R., RUNDEL, P. \& CORADIN, V.T.R. 2005. Leaf functional traits of Neotropical savanna trees in relation to seasonal water deficit. Trees Structure and Function 19:326-335.

GARTEN JUNIOR, C. T. 1976. Correlations between concentrations of elements in plants. Nature 261:686-688.

HARIDASAN, M. 1987. Distribution and mineral nutrition of aluminium accumulating species in different plant communities of the cerrado region of central Brazil. In La Capacidad Bioproductiva de Sabanas. (J.J. San José \& R. Montes, eds.) I.V.I.C., Caracas, Venezuela. p.309348.

HARIDASAN, M. 1994. Solos do Distrito Federal. In Cerrado: Caracterização, ocupação e perspectivas - o caso do Distrito Federal. (M. Novaes-Pinto, ed.) 2 ${ }^{\mathrm{a}}$ ed. Editora Universidade de Brasília/SEMATEC, Brasília. p.321-344.

HARIDASAN, M. \& ARAÚJO, G.M. 2005. Perfil nutricional de espécies lenhosas de duas florestas semidecíduas em Uberlândia, MG. Revista Brasileira de Botânica 28:295303.

HOFFMANN, W.A., FRANCO, A.C., MOREIRA, M.Z., \& HARIDASAN, M. 2005. Specific leaf area explains differences in leaf traits between congeneric savanna and forest trees. Functional Ecology 19:932-940.

LENZA, E. \& KLINK, C.A. 2006. Comportamento fenológico de espécies lenhosas em um cerrado sentido restrito de Brasília, DF. Revista Brasileira de Botânica 29:627-638.

LOPES, A.S. \& COX, F.R. 1977. A survey of the fertility status of surface soils under "cerrado" vegetation in Brazil. Soil Science Society of America Journal 41:742-747.

MARIMON JÚNIOR, B.H. \& HARIDASAN, M. 2005. Comparação da vegetação arbórea e características edáficas de um cerradão e um cerrado sensu stricto em áreas adjacentes sobre solo distrófico no leste de Mato Grosso, Brasil. Acta Botanica Brasílica 19:913-926. 
MEZIANE, D. \& SHIPLEY, B. 2001. Direct and indirect relationships between specific leaf area, leaf nitrogen and leaf gas exchange. Effects of irradiance and nutrient supply. Annals of Botany 88:915-927.

MONTES, R. \& MEDINA, E. 1977. Seasonal changes in nutrient content of leaves of savanna trees with different ecological behavior. Geologia y Ecologia Tropical 4:295-307.

NARDOTO, G.B., BUSTAMANTE, M.M.C., PINTO, A.S. \& KLINK, C.A. 2006. Nutrient use efficiency at ecosystem and species level in savanna areas of Central Brazil and impacts of fire. Journal of Tropical Ecology 22:191-201.

PERES, J.R.R., SUHET, R., VARGAS, M.A.T. \& DROSDOWICZ, A. 1983. Litter production in two cerrado vegetations in Brazil. Pesquisa Agropecuária Brasileira 18:1037-1043.

POORTER, H. \& GARNIER, E. 1999. Ecological significance of inherent variation in relative growth rate and its components. In Handbook of Functional Plant Ecology (F.I. Pugnaire \& F. Valladares, eds.). Marcel Dekker, New York. p. 81-120.

RECOR 2007. http://www.recor.org.br/Estacao/estacao.html acesso em 17/06/2007.

REICH, P.B. 1993. Reconciling apparent discrepancies among studies relating life span, structure and function of leaves in contrasting plant life forms and climates: "the blind men and the elephant retold". Functional Ecology 7: 721-725.

REICH, P.B., WALTERS, M.B \& ELLSWORTH, D.S. 1992. Leaf lifespan in relation to leaf plant and stand characteristics among diverse ecosystem. Ecological monographs 62: 365-392.

REICH, P.B., WALTERS, M.B. \& ELLSWORTH, D.S. 1997. From tropics to tundra: Global convergence in plant functioning. Proceedings of the National Academy of Science 94:13730-13734.
SAS 2006. The SAS - System for Windows, Release 9.3, SAS Inst. Inc., Cary, NC, USA.

SILVA, D.A. 2001. Comparação de características foliares de espécies lenhosas entre cerrado denso, cerrado sensu stricto e campo sujo na Reserva Ecológica do IBGE, Brasília (DF). Dissertação de mestrado, Universidade de Brasília, Brasília.

SILVA JÚNIOR, M.C. 2005. 100 árvores do cerrado - guia de campo. Editora Brasília: Rede de Sementes do Cerrado. Brasília.

VILE, D. GARNIER, E., SHIPLEY, B., LAURENT, G., NAVAS, M.L., ROUMET, C., LAVOREL, S., DI'AZ, S., HODGSON, J., LLORET, F., MIDGLEY, G.F., POORTER, H., RUTHERFORD, M.C., WILSON, P.J. \& WRIGHT, I.J. 2005. Specific leaf area and dry matter content estimate thickness in laminar leaves. Annals of Botany 96:11291136.

VILLAR, R. \& MERINO, J. 2001. Comparison of leaf construction costs in woody species with differing leaf life-spans in contrasting ecosystems. New Physiologist 151:213-226.

VILLAR, R., ROBLETO, J.R., JONG, Y. \& POORTER, H. 2006. Differences in construction costs and chemical composition between deciduous and evergreen woody species are small as compared to differences among families. Plant, Cell and Environment 29:1629-1643.

VILLELA, D.M. \& LACERDA, L.D. 1992. Dinâmica de elementos minerais em folhas de duas espécies arbóreas do cerrado. Revista Brasileira de Botânica 52:151-160.

WESTOBY, M., WARTON, D. \& REICH, P.B. 2000. The time value of leaf area. The American Naturalist 155:649-656.

WRIGHT, I.J., REICH, P.B. \& WESTOBY, M. 2001. Strategy shifts in leaf physiology, structure and nutrient content between species of high-and-low-rainfall and high and low-nutrient habitats. Functional Ecology 15:423-434. 